\title{
(6) OPEN ACCESS \\ Chinese military medical teams in the Ebola outbreak of Sierra Leone
}

\author{
Yinying Lu, ${ }^{1} \mathrm{G}$ Rong, ${ }^{1} \mathrm{~S} \mathrm{P} \mathrm{Yu},{ }^{2} \mathrm{Z}$ Sun, ${ }^{1} \mathrm{X}$ Duan, ${ }^{1} \mathrm{Z}$ Dong, ${ }^{1} \mathrm{H} \mathrm{Xia},{ }^{1} \mathrm{~N}$ Zhan, ${ }^{1} \mathrm{C}$ Jin, ${ }^{1}$ \\ $\mathrm{J} \mathrm{Ji}{ }^{1} \mathrm{H}$ Duan $^{1}$
}

${ }^{1}$ The 302 Hospital of Chinese Army, Beijing, China ${ }^{2}$ Health Services Center, Chinese Academy of Military Medical Sciences, Beijing, China

\section{Correspondence to}

Col Huijuan Duan, The 302 Hospital of Chinese Army, 100 Xi-Si-Huan Middle Road, Beijing 100039, China; Huijuanduan_302@126.com

$Y L$ and GR contributed equally.

Received 16 September 2015 Revised 21 November 2015 Accepted 25 November 2015 Published Online First

7 January 2016

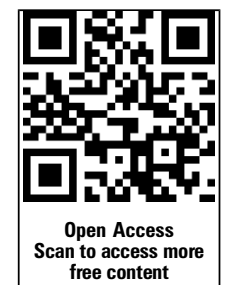

CrossMark

To cite: $L u Y$, Rong $G$ Yu SP, et al. J R Army Med Corps 2016;162:198-202.

\section{ABSTRACT}

The 2014-2015 Ebola virus disease (EVD) epidemic in West Africa was the largest in history. The three most affected countries, Guinea, Liberia and Sierra Leone, have faced enormous challenges in controlling transmission and providing clinical care for patients with EVD. The Chinese government, in response to the requests of the WHO and the governments of the affected countries, responded rapidly by deploying Chinese military medical teams (CMMTs) to the areas struck by the deadly epidemic. A total of three CMMTs, comprising 115 military medical professionals, were rotationally deployed to Freetown, Sierra Leone to assist with infection prevention and control, clinical care and health promotion and training. Between 1 October 2014 and 22 March 2015, the CMMTs in Sierra Leone admitted and treated a total of 773 suspected and 285 confirmed EVD cases. Among the 285 confirmed cases, $146(51.2 \%)$ patients survived after treatment. In addition, the CMMTs maintained the record of zero infections among healthcare workers and zero cross-infections between quarantined patients. In this manuscript, we aim to give an overview of the mission, and share our best practices experience on predeployment preparedness, EVD holding and treatment centre building and EVD case management.

\section{INTRODUCTION}

The 2014-2015 Ebola virus disease (EVD) epidemic in West Africa was the largest in history. The three most affected countries, Guinea, Liberia and Sierra Leone, have faced enormous challenges in controlling transmission and providing clinical care for patients with EVD. On 8 August 2014, the WHO officially announced that the current EVD outbreak had become a public health emergency and urgently called upon the international community to provide medical aid. ${ }^{1}$ In response to this call, the Chinese government activated its civilmilitary coordinating disaster rescue mechanism by deploying Chinese military medical teams (CMMTs) to Sierra Leone and Liberia, two of the three most affected countries. From 15 September 2014 to 20 March 2015, three CMMTs consisting of 115 military medical professionals, including doctors, nurses and public health experts from the 302 hospital, a military hospital specific for infectious diseases (IDs), were rotationally deployed to Freetown, Sierra Leone to provide clinical care, infection control and health promotion.

This manuscript gives an overview of the rescue mission of the CMMTs in Sierra Leone, and shares our best practice experiences on predeployment
Key messages

The 2014-2015 Ebola virus disease (EVD) epidemic in West Africa was the largest recorded in history.

- Three Chinese military medical teams (CMMTs) were deployed to Sierra Leone to assist with infection prevention and clinical care of EVD.

- The CMMTs in Sierra Leone admitted and treated a total of 773 suspected and 285 confirmed EVD cases.

- The CMMTs in Sierra Leone admitted a total of 773 suspected and 285 confirmed EVD cases. Among the 285 confirmed cases, 146 survived after treatment.

preparedness, EVD holding and treatment centre (HTC) building and EVD case management.

\section{PREDEPLOYMENT PREPAREDNESS}

A CMMT in Sierra Leone was designed to have four functional units: organisational administration, medical care, infection prevention and control (IPC) and logistics. Team members were selected accordingly from the 302 Hospital of the Chinese Army, a military hospital specialising in IDs. Doctors and nurses were required to be middle or senior level with excellent clinical skills, and most of them had participated in rescue missions during the 2003 SARS outbreak, the 2008 Wenchuan earthquake or the earthquake in Haiti in 2010. Overall, there were 28 doctors, 50 nurses, 12 IPC officers and 25 logistical personnel, and the specialties among the recruited doctors included ID, surgery, critical-care medicine, radiology and laboratory medicine.

Except for members of the first-deployed CMMT, all other members received a 4-week, fulltime training course covering language, local culture, EVD virological knowledge, personal protective equipment (PPE) usage, EVD clinical care, facility sterilisation and public sanitation. The training course was administered in a three-step process and the content was based on documents released by the WHO and the Chinese and Sierra Leonean governments. $^{2-4}$ The first and second steps were theoretical study and PPE usage, respectively. In the third step, the trainees received training in EVD clinical care in a ward designed to simulate a reallife EVD ward in Sierra Leone, with features such as a highly humid environment and an average 
temperature of $30^{\circ} \mathrm{C}-34^{\circ} \mathrm{C}$. To minimise the risk of EVD exposure, all members had to pass an examination at the end of each step before progressing.

\section{BUILDING THE EVD HTC}

Members of the first CMMT received the order to embark on the rescue mission on 13 September 2014 and arrived in Sierra Leone 4 days later. In Freetown, they rebuilt the Jui Government Hospital and converted it into a specialised EVD HTC with a 40-bed capacity, which could be expanded to 78 beds, within the first week of their deployment. The Sierra Leone government then renamed the hospital as China-Sierra Leone Friendship Hospital. The construction of the hospital was based upon the standards required by the Chinese ID hospital architecture design specification, the Chinese ID hospital construction standards and the established WHO practices guidelines. $^{2-5}$ The interior layout structure of the China-Sierra Leone joint EVD HTC is shown in Figure 1.

All other preparation work including ward decoration, equipment setting, local staff training and drafting of standard operation procedures (SOPs) for EVD treatment were completed in the following 2 weeks. On 1 October 2014, the China-Sierra Leone joint EVD HTC was fully functional, and soon became a major international medical aid facility in the Freetown area.

\section{CLINICAL MANAGEMENT OF EVD CASES}

Timely patient quarantine, early diagnosis and effective care are key factors for the termination of EVD transmission. According to the SOP, for EVD case screening (Figure 2), a rapid assessment of every incoming patient with EVD-like symptoms at the triage area of the EVD HTC was performed. ${ }^{6}{ }^{7}$ Suspected cases were immediately admitted to the quarantine wards. In order to confirm the diagnosis of EVD, a blood sample from each patient was tested by an Ebola-virus-specific PCR test in a mobile clinical laboratory with results returned within $24 \mathrm{~h}$ of an individual being quarantined. Confirmed EVD cases were transferred to the treatment ward within the second $24 \mathrm{~h}$ of quarantine while those with negative results were discharged. For patients with symptoms of less than $72 \mathrm{~h}$ or who were younger than 12 years of age, a prolonged period of observation was maintained until EVD was ruled out by a second PCR examination $48 \mathrm{~h}$ later, even if they manifested negative results in the first round PCR test.

To standardise the management of EVD, the CMMTs issued a series of SOPs that included 68 categories and 243 separate items. These SOPs standardised every detail of EVD management including staff training, work shifts, ward rounds, triage processes, patient history collection, medical document writing, nursing care, medicine provision, IPC, waste disposal and emergency disposal. The CMMTs also developed SOPs for PPE use, EVD case management, EVD nursing and hospital infection control in the China-Sierra Leone joint EVD HTC.

The CMMTs provided comprehensive and stratified treatments to EVD cases. Upon confirmation, all patients were placed into one of three categories (mild, medium and severe) by a group of senior doctors according to the severity of the illness. The parameters considered were age, the presence of hypovolemic shock or coma or organ failure, baseline conditions and complications and the results of radiological and laboratory examinations. The most deadly pathophysiological change caused by EVD was hypovolemic shock caused by severe vomiting and diarrhoea or internal bleeding; thus, the primary principle of our therapeutic strategy was to provide adequate fluid resuscitation and to correct electrolyte abnormalities. This was generally achieved by the administration of rehydration fluid orally in mild and medium category patients or intravenously in severe ill patients.

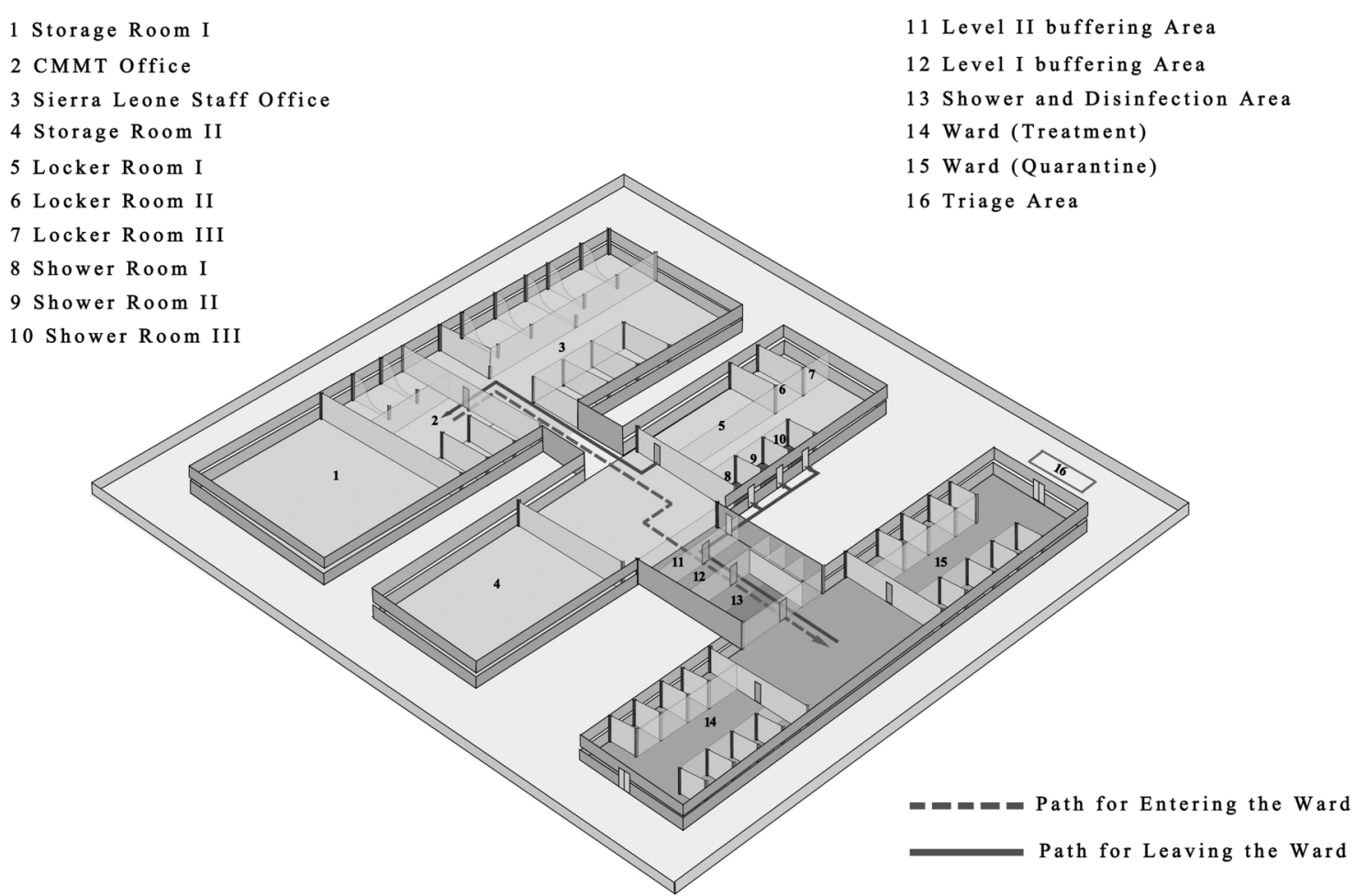

Figure 1 The indoor structure of the China-Sierra Leone joint Ebola virus disease holding and treatment centre. CMMT, Chinese military medical team. 


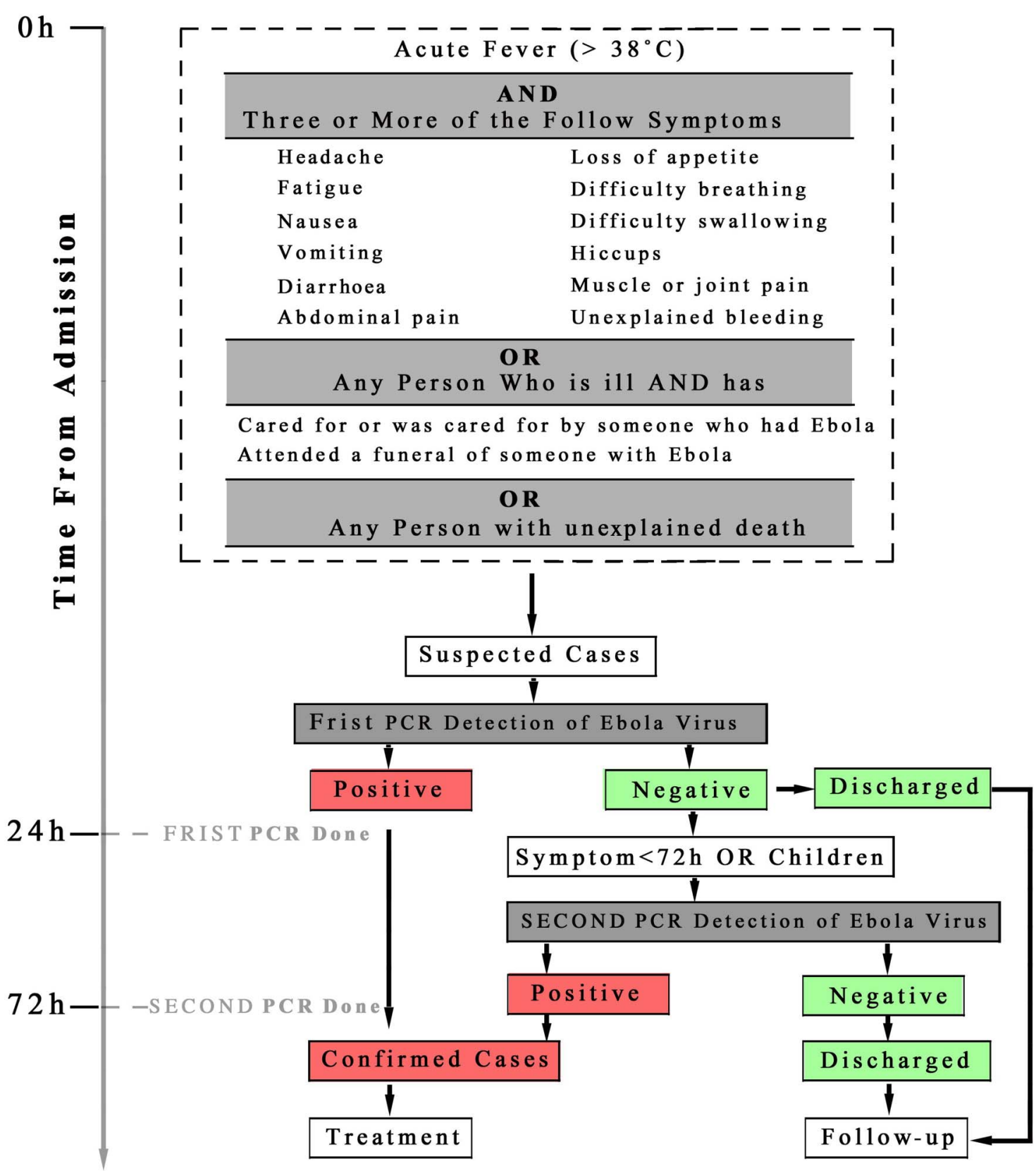

Figure 2 The flow chart of Ebola virus disease (EVD) case screening in the China-Sierra Leone joint EVD holding and treatment centre.

In addition, treatment aimed to protect or restore the function of vital organs such as the brain, kidney and liver, and to provide adequate energy and nutrition as well as any other appropriate symptom-relief treatments. ${ }^{2}$

Approximately $15 \%$ of our patients were children under the age of 12 , and in addition to the regular treatments, additional supportive treatments were also introduced to paediatric EVD cases-zinc supplementation was given to children who had suffered from diarrhoea for more than 10 days and vitamins $A$ and $\mathrm{K}$ were provided to all children under five and children with haemorrhage, respectively. ${ }^{2}$

Between 1 October 2014 and 22 March 2015, the ChinaSierra Leone joint EVD HTC admitted and treated 773 suspected EVD cases and 285 confirmed EVD cases; among the 285 confirmed cases, 146 (51.2\%) survived and 139 died (48.8\%). The CMMT's clinical performance did not rank well at the early stage of the mission, as measured by a weekly clinical quality report issued by the Ministry of Health, Sierra Leone; however, after overcoming some early challenges, the CMMT caught up and scored well on the weekly report comparable with other international agencies.

There were zero infections among healthcare workers (HCWs) and no cross-infections between quarantined patients for 171 days. This achievement was attributed to six key strategies (Box 1).

\section{EPIDEMIOLOGICAL AND VIROLOGICAL DATA COLLECTION AND PATIENT FOLLOW-UP}

As the China-Sierra Leone joint EVD HTC was the frontline in the battle to combat the widespread emergence of the Ebola virus, it was vitally important to collect real-time clinical data for decision makers' use when calibrating their anti-EVD policy; 
Box 1 Key features contributing to the Ebola virus disease (EVD) holding and treatment centre (HTC) zero infection rate

- All the Chinese military medical team (CMMT) personnel were experienced doctors and nurses specifically trained in infectious diseases who were used to routinely protecting themselves and patients from infection. Each member took part in a 4-week, full-time training course, and only those who passed a final exam were permitted to join the CMMT. This was a well-disciplined and organised military medical unit, and each member was ordered to take all steps needed to avoid healthcare worker (HCW) infection during the entire mission.

- The HCWs in the Chinese-Sierra Leone joint EVD HTC were divided into small groups (three to four people), and the group members worked together to protect each other and to avoid unexpected exposure. In addition, via surveillance cameras, the IPC team members monitored the whole HTC $24 / 7$, and warnings were issued when behaviours deemed to pose a risk of EVD transmission was observed.

- Patients were quarantined in separate rooms whenever possible; when not possible, patients from the same family or with the same exposure history or with similar disease severity were placed in the same room.

- Inside the quarantine rooms, the beds were at least $1.5 \mathrm{~m}$ apart and physical contact between the patients was strictly prohibited. The IPC group members patrolled the ward every hour and continuously monitored all patients through the surveillance cameras.

- Each patient had their own PPE and other supplies.

- To reduce the duration of quarantine and accelerate the recycling of rooms, all PCR results had to be returned within $24 \mathrm{~h}$.

the CMMTs established a clinical data management group (CDMG) to collect, analyse and report our EVD data and to follow up on the discharged cases. Patients discharged with a negative diagnosis were to be followed up weekly by telephone for 4 weeks. Those with no reported EVD-related death or rehospitalisation within 4 weeks of discharge, bearing in mind that the incubation time of EVD is 21 days, were defined as clear of nosocomial infection. The names of those patients who could not be reached by telephone were searched for in the central database at the EVD command centre of Sierra Leone to rule out EVD-related death or rehospitalisation within 4 weeks of discharge, to provide definitive evidence of nosocomial infection in all of the 773 suspected EVD cases.

A specifically designed unified EVD case report form held on a digital database coupled with the China mobile laboratory testing team in Sierra Leone were able to successfully monitor the genetic diversity of the Ebola virus in real time. By analysing the information gathered from 175 full-length Ebola virus genome sequences from five districts in Sierra Leone, their recent study revealed a sharp increase in the genetic diversity of the virus since $2014 .^{8}$

By 22 March 2015, the third and last CMMT in Sierra Leone had successfully completed its mission. Two days before it left, the Sierra Leone Ministry of Health and Sanitation, for the first time since the EVD epidemic started, declared no newly reported cases. ${ }^{9}$ Although there were 28183 confirmed cases and 11306 deaths reported in West Africa during the outbreak, ${ }^{10}$ the international community finally saw hope of victory in the battle against Ebola. The CMMTs have made important contributions to the success of this battle through their quick response, professional EVD management and robust support of the local health system.

\section{CONCLUSIONS}

This was the first time that the Chinese military medical forces have performed a humanitarian mission as an independent unit; it had no previous experience in hospital building, foreign staff training, long-term overseas logistic support or international cooperation. During the mission, the teams obtained enormous valuable experience in preventing and controlling this public health crisis, but also faced tremendous challenges. For example, for some time, communication between the CMMTs and the local HCWs and health administrative officers was poor because of language and cultural differences between China and Sierra Leone. This may be the main reason for the relatively poor ranking of Jui Hospital in the early stages of the mission. Although the language and cultural differences were quickly overcome, they could be a substantial barrier in future missions. In addition, as the Chinese Army does not have an overseas logistic chain, securing equipment and supplies from the homeland is an additional challenge for CMMTs. In the present case, the first CMMT had to ensure it had enough medical supplies for 2 months, while the medical supplies for the second and third CMMTs were shipped by sea 2 months before the teams set off, and they purchased most of their food supplies from the local market.

The preservation of human life should be an urgent concern of all governments when countries are confronted with a disaster like the 2014 EVD outbreak. The Chinese nation and its Army are ready to contribute more actively to the international community when humanitarian issues arise and it is likely that increasing numbers of Chinese medical teams, either military or civilian, will be seen in the field fighting against natural disasters in the future.

Contributors YL, GR and SPY substantial contributed to the conception and design of the work and revised the draft critically for important intellectual content. Each author has contributed to sections of the manuscript. Each author has edited the manuscript. JJ and HD get the final approval of the version published and are the guarantors.

\section{Competing interests None declared.}

Provenance and peer review Not commissioned; externally peer reviewed.

Open Access This is an Open Access article distributed in accordance with the Creative Commons Attribution Non Commercial (CC BY-NC 4.0) license, which permits others to distribute, remix, adapt, build upon this work non-commercially, and license their derivative works on different terms, provided the original work is properly cited and the use is non-commercial. See: http://creativecommons.org/ licenses/by-nc/4.0/

\section{REFERENCES}

1 Team WER. Ebola virus disease in West Africa - the first 9 months of the epidemic and forward projections. N Engl J Med 2014;371:1481-95.

$2 \mathrm{MOHs}$ of Sierra Leone and WHO. Clinical Management of Patients in the Ebola Treatment Centers and other care centers in Sierra Leone: A Pocket Guide. https://extranet.who.int/ebolafmt/sites/default/files/documents/Sierra Leone Ebola Treatment Centre pocket guide 15 Dec 2014(1).pdf (accessed 15 Sep 2015).

3 Personal protective equipment in the context of filovirus disease outbreak Response, rapid advice guideline. http://apps.who.int/iris/bitstream/10665/137410/ 1/WHO_EVD_Guidance_PPE_14.1_eng.pdf (accessed 15 Sep 2015).

4 The Minister of Health of the People's Republic of China. Clinical strategy for the diagnosis and treatment of Ebola hemorrhagic fever. Infect Dis Info 2014;20:58-9. 


\section{Original article}

5 Ministry of Construction of People's Republic of China. National Standard of infectious disease hospital architecture (Standard code: GB50849). China Planning Press, 2015.

6 World Health Organization. Case definition recommendations for Ebola or Marburg virus diseases. http://www.who.int/csr/resources/publications/ebola/ ebola-case-definition-contact-en.pdf (accessed 15 Sep 2015).

7 Tattevin $\mathrm{P}$, Durante ME, Massaquoi $\mathrm{M}$ et al. Does this patient have Ebola virus disease? Intensive Care Med 2014;40:1738-41.
8 Tong YG, Shi WF, Liu D, et al. Genetic diversity and evolutionary dynamics of Ebola virus in Sierra Leone. Nature 2015:524:93-6.

9 Sierra Leone Ministry of Health and Sanitation. EBOLA OUTBREAK UPDATES 20 March 2015. http://health.gov.s//wp-content/uploads/2015/03/ Ebola-Update-March-20-2015.pdf (accessed 15 Sep 2015).

10 WHO. EBOLA SITUATION REPORT, 9 September 2015. http://apps.who.int/iris/ bitstream/10665/184271/1/ebolasitrep_9Sept2015_eng.pdf (accessed 15 Sep 2015). 